\title{
ENERGETIC PARTICLE OBSERVATIONS AND PROPAGATION IN THE THREE-DIMENSIONAL HELIOSPHERE DURING THE 2006 DECEMBER EVENTS
}

\author{
O. E. Malandraki ${ }^{1,2}$, R. G. Marsden ${ }^{2}$, D. Lario ${ }^{3}$, C. Tranquille ${ }^{2}$, B. Heber ${ }^{4}$, R. A. Mewaldt ${ }^{5}$, C. M. S. Cohen ${ }^{5}$, \\ L. J. Lanzerotti ${ }^{6,7}$, R. J. Forsyth ${ }^{8}$, H. A. Elliott ${ }^{9}$, I. I. Vogiatzis $^{10}$, And A. Geranios ${ }^{11}$ \\ ${ }^{1}$ Institute for Astronomy and Astrophysics, National Observatory of Athens, Metaxa \& Vas. Pavlou str., Palaia Pedeli 15236, Athens, Greece; omaland@astro.noa.gr \\ ${ }^{2}$ Research and Scientific Support Department of ESA, ESTEC, P.O. Box 299, 2200 AG Noordwijk, The Netherlands \\ ${ }^{3}$ Applied Physics Laboratory, Johns Hopkins University, 11100 Johns Hopkins Road, Laurel, MD, USA \\ ${ }^{4}$ Christian-Albrechts-Universität Kiel, Leibnistrasse 11, Kiel, D-24118, Germany \\ ${ }^{5}$ Space Radiation Laboratory, California Institute of Technology, Pasadena, CA 91125, USA \\ ${ }^{6}$ Center for Solar-Terrestrial Research, New Jersey Institute for Technology, 323 Martin Luther King Boulevard, 101 Tiernan Hall, Newark, NJ 07102-1982, USA \\ ${ }^{7}$ Bell Laboratories, Alcatel-Lucent, NJ, USA \\ ${ }^{8}$ The Blackett Laboratory, Imperial College of Science and Technology London, Imperial College London, South Kensington campus, London, SW7 2AZ, England \\ ${ }^{9}$ Space Science and Engineering, Southwest Research Institute, San Antonio, TX 78238, USA \\ ${ }^{10}$ Space Science Laboratory, Democritus University of Thrace, Xanthi, Greece \\ ${ }^{11}$ Nuclear and Physics Department, University of Athens, Panepistimioupoli, Ilissia, 15771 Athens, Greece \\ Received 2009 February 14; accepted 2009 August 25; published 2009 September 23
}

\begin{abstract}
We report observations of solar energetic particles obtained by the HI-SCALE and COSPIN/LET instruments onboard Ulysses during the period of isolated but intense solar activity in 2006 December, in the declining phase of the solar activity cycle. We present measurements of particle intensities and also discuss observations of particle anisotropies and composition in selected energy ranges. Active Region 10930 produced a series of major solar flares with the strongest one (X9.0) recorded on December 5 after it rotated into view on the solar east limb. Located over the South Pole of the Sun, at $>72^{\circ} \mathrm{S}$ heliographic latitude and 2.8 AU radial distance, Ulysses provided unique measurements for assessing the nature of particle propagation to high latitudes under near-minimum solar activity conditions, in a relatively undisturbed heliosphere. The observations seem to exclude the possibility that magnetic field lines originating at low latitudes reached Ulysses, suggesting either that the energetic particles observed as large solar energetic particle (SEP) events over the South Pole of the Sun in 2006 December were released when propagating coronal waves reached high-latitude field lines connected to Ulysses, or underwent perpendicular diffusion. We also discuss comparisons with energetic particle data acquired by the STEREO and Advanced Composition Explorer in the ecliptic plane near 1 AU during this period.
\end{abstract}

Key words: Sun: coronal mass ejections (CMEs) - Sun: flares - Sun: magnetic fields - Sun: particle emission solar wind

\section{INTRODUCTION}

Launched in 1990 October, the joint ESA/NASA Ulysses mission has explored the previously uncharted third dimension of our solar system. During its operational lifetime of almost 19 years, Ulysses completed three orbits carrying it over both poles of the Sun.

Although the Sun was again close to its activity minimum during the recently completed third polar orbit of Ulysses, solar activity has been more prevalent during the declining phase of Solar Cycle 23 (McKibben et al. 2005; Malandraki et al. 2007, 2008) than was the case in the declining phase of the 22nd solar cycle, when the first polar passes occurred (1994/1995) (e.g., Lario \& Pick 2008). In 2006 December, an unexpected rise of solar activity occurred. Active Region 10930 near the Sun's equator began producing X-class flares almost as soon as it rotated onto the visible hemisphere of the Sun on December 5 (see Table 1). The Ulysses spacecraft was then luckily over the South Pole of the Sun, at $>72^{\circ}$ southern heliographic latitude and a heliocentric distance of $\sim 2.8 \mathrm{AU}$. This provides a unique opportunity in the history of the Ulysses mission to study a large solar energetic particle (SEP) event at high heliolatitudes $\left(>70^{\circ} \mathrm{S}\right)$ near solar minimum, with a relatively quiet and stable interplanetary structure in the heliosphere. In this work, we present in detail energetic particle observations $(77 \mathrm{keV}$ to $\sim 20 \mathrm{MeV}$ ions and $35-315 \mathrm{keV}$ electrons) obtained during this unique event by Ulysses and discuss their implications for particle propagation to high solar latitudes (see Heber et al. 2008 for a discussion of higher energy particle observations by Ulysses during this period). The observed events are also compared with high-latitude measurements obtained previously by Ulysses close to solar maximum. Furthermore, comparisons with data acquired by the STEREO and Advanced Composition Explorer (ACE) spacecraft near the ecliptic plane are discussed.

\section{INSTRUMENTATION}

In this paper, we use energetic particle data from the Cosmic Ray and Solar Particle Investigation (COSPIN)/Low Energy Telescope (LET) instrument (Simpson et al. 1992) and the Heliospheric Instrument for Spectra, Composition, and Anisotropy at Low Energies (HI-SCALE) instrument (Lanzerotti et al. 1992) onboard Ulysses. Magnetic field observations from the Ulysses magnetometer (VHM-FGM; Balogh et al. 1992) and solar wind measurements from the Ulysses Solar Wind Plasma experiment (SWOOPS; Bame et al. 1992) are also used. The COSPIN/ LET instrument records, the fluxes, and the composition of solar energetic particles and low-energy cosmic ray nuclei from hydrogen up to iron over a range of energies from $\sim 1 \mathrm{MeV} / \mathrm{n}$ to $\geqslant 50 \mathrm{MeV} / \mathrm{n}$. Ion data from the LEMS120 telescope (Low Energy Magnetic Spectrometer) and differential intensities of 35-315 keV magnetically deflected electrons (DE) measured by the WART B detector head of the HI-SCALE experiment 
Table 1

Significant Solar Events (M3 or Greater) Reported in 2006 December

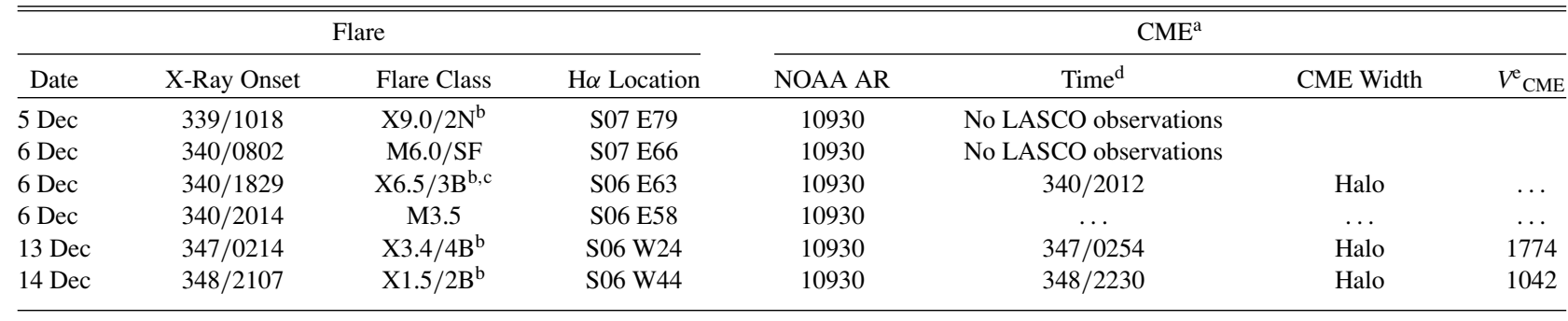

Notes.

${ }^{a}$ CME classification and parameters extracted from the SOHO/LASCO CME catalog at http://cdaw.gsfc.nasa.gov//.

b Flare associated with intense metric type II radio burst (as reported in the SEC Preliminary Report and Forecast of Solar Geophysical Data published online at http://www.swpc.noaa.gov/weekly/index.html by the NOAA/SED and the AFWA).

c Moreton wave observed to flow out of flare (http://spaceweather.com/index.html).

${ }^{\mathrm{d}}$ First appearance in the $\mathrm{C} 2$ coronagraph ( $>1.5$ solar radii).

e Plane of sky projected CME speed in $\mathrm{km} \mathrm{s}^{-1}$.

are also utilized. The WART B detector head has a geometrical factor of $\sim 0.05 \mathrm{~cm}^{2}$ sr. We also use electron intensities and their angular distributions in the energy range 178-290 keV detected by the sunward looking telescope LEFS60 (Low-Energy Foil Spectrometer) of the HI-SCALE experiment, which has a larger geometrical factor $\left(\sim 0.48 \mathrm{~cm}^{2} \mathrm{sr}\right)$. Energetic particle data measured in the ecliptic plane near $1 \mathrm{AU}$ were obtained from the Low Energy Telescope (LET; Mewaldt et al. 2008) of the in situ measurements of particles and CME transients investigation (IMPACT; Luhmann et al. 2008) onboard the STEREO spacecraft and the Electron, Proton, Alpha Monitor (EPAM) instrument onboard the $A C E$ spacecraft (Gold et al. 1998). EPAM was adapted from the flight-spare unit of the Ulysses/HI-SCALE instrument.

\section{OBSERVATIONS AND DATA ANALYSIS}

\subsection{Solar Activity}

Table 1 contains a list of the major solar events and their characteristics for 2006 December. M3 or greater class flares and associated coronal mass ejections (CMEs) are listed. The solar flares are as reported in the solar geophysical data (http://sgd.ngdc.noaa.gov/sgd/jsp/solarindex.jsp) whereas, when available, CME identification and parameters were obtained from the Solar and Heliospheric Observatory/Large Angle and Spectrometric Coronagraph (SOHO/LASCO) CME catalog compiled by S. Yashiro and G. Michalek (available at http://cdaw.gsfc.nasa.gov). After it rotated into view on the east limb, Active Region 10930 began producing a series of Xand M-class flares. The four X-class flares during this period of isolated but intense solar activity are highlighted using the bold-faced type in Table 1. The strongest one was an X9.0 solar flare recorded on December 5 at S07 E79 as viewed from the Earth. On December 6, region 10930 produced an X6.5 flare, and as it rotated across the solar disk, two more X-class flares were observed on December 13 and 14, both in the western hemisphere of the Sun and both associated with fast halo CMEs. All X-ray flares of this period were associated with particularly intense metric type II radio bursts, indicative of propagating coronal shock waves at the Sun (e.g., Vainio \& Khan 2004; Mann 2006). Furthermore, a rare Moreton waveaka "solar tsunami" was observed to flow out of the X6.5 flare (http://spaceweather.com/index.html).

\subsection{High-latitude Observations}

Figure 1 shows the position of Ulysses, then at a heliocentric distance of $\sim 2.8 \mathrm{AU}$ and at $>72^{\circ}$ southern heliographic latitude, with respect to the fixed Sun-Earth line, projected onto the solar equatorial plane as viewed from the north (top) and on a plane perpendicular to the solar equator as viewed from the Earth toward the Sun (bottom) on DOY 339 (December 5) (left) and DOY 347 (December 13) (right). The ideal Archimedes spiral magnetic field lines connecting Ulysses to the Sun are also shown, calculated using the measured solar wind speed at Ulysses on the two days. The negative values in red correspond to locations east of the central meridian. On December 5, there was an angular separation of $70^{\circ}$ in longitude between the nominal Ulysses magnetic footpoint and the X9.0 flare location. The great circle angular separation between the spacecraft footpoint and the flare was $\sim 77^{\circ}$. This separation was $\sim 135^{\circ}$ for the Earth. Subsequently, as the Sun rotated, Ulysses became more poorly connected, while the Earth became better connected to the flare sites.

In the top panel of Figure 2, an overview of the spin-averaged energetic ion intensities as measured by the Ulysses/HI-SCALE LEMS120 telescope in the energy range 77-1123 keV and the Ulysses/COSPIN/LET telescope in the energy range 1.8$19 \mathrm{MeV}$ in 2006 December is presented. The second panel presents the integral rate of higher energy $(>50 \mathrm{MeV})$ ions as measured by COSPIN/LET. The vertical arrows in this panel indicate the occurrence of the flares in Table 1. Longitude information is given for the four X-class flares observed. Magnetically deflected near-relativistic electrons (DE) as measured by the WART B detector head of the HI-SCALE experiment for the same period are shown in the third panel. The panels below present solar wind velocity and interplanetary magnetic field (IMF) magnitude, meridional angle, and azimuth angle in the Ulysses Radial-Targential-Normal (RTN) coordinate system (Forsyth et al. 1995). The radial solar distance and the heliographic latitude of the spacecraft are shown at the top of Figure 2. The HI-SCALE ion channels may be contaminated at the beginning of large SEP events when many electrons arrive promptly, well before the slower ions can reach the spacecraft (e.g., Lario et al. 2004). By comparing electron intensities with low-energy ion intensities and COSPIN/LET intensities with HI-SCALE/ LEM120 intensities in 2006 December, we conclude that the $<1 \mathrm{MeV}$ ion channels of the LEMS120 were contaminated in 

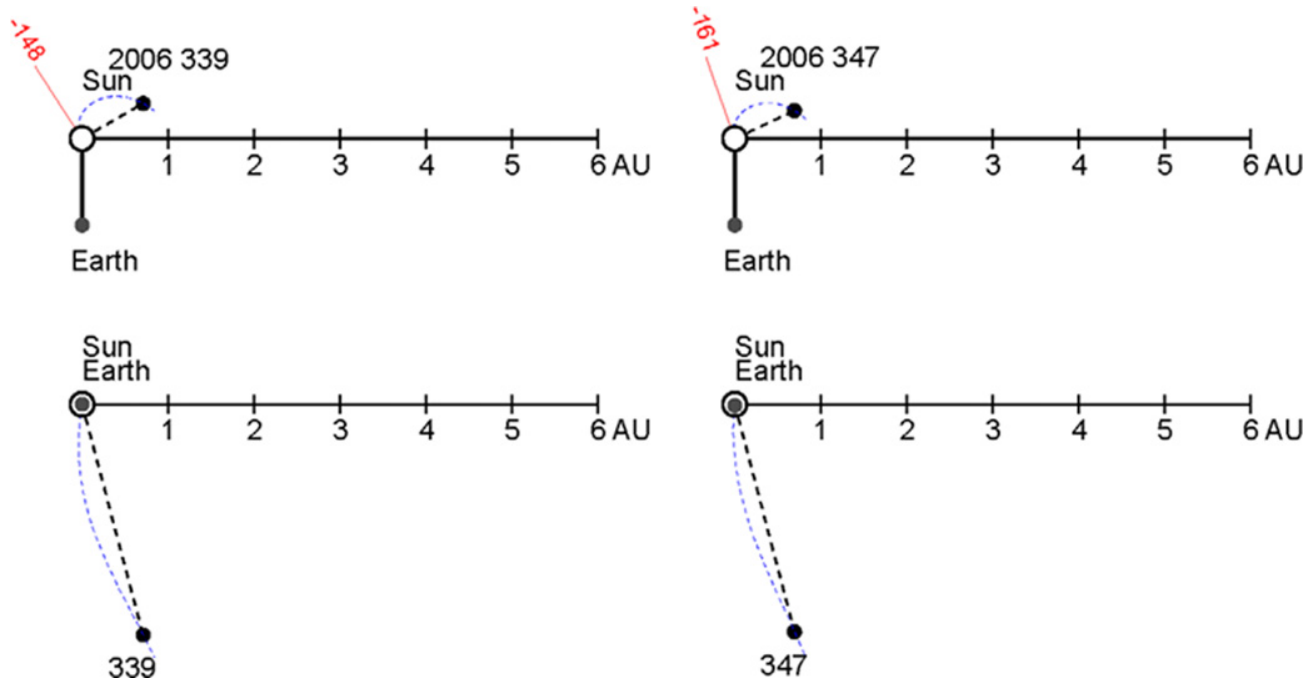

Figure 1. Position of Ulysses with respect to the fixed Sun-Earth line on December 5 (DOY 339) and 13 (DOY 347 ); see the text.

the rising phase of the SEP event by electrons bypassing the magnetic deflection system. We have indicated this period by dotted traces in Figure 2. The solid vertical lines in Figure 2 indicate the time of passage of three forward $(\mathrm{F})$ shocks during this period. The shocks are obvious from the abrupt increases in the solar wind speed and magnetic field magnitude. No associated driver ejecta signatures were detected during this period (Ebert et al. 2009; Liu et al. 2008). This constitutes a unique observation by Ulysses at high latitudes.

As is evident from Figure 2, major SEP events were observed by Ulysses in response to the rise of solar activity in 2006 December. These events were observed when Ulysses was immersed in the high speed solar wind flow emanating from the southern polar coronal hole (McComas et al. 2008). As discussed below, the shocks observed are most likely related to Active Region 10930 and the associated X-class flares. The shocks arrive at Ulysses on DOY 344 (December 10), 345 (December 11), and 351 (December 17) so the delays with respect to the X-ray flare onsets are all about five days, consistently implying shock speeds of the order of $1000 \mathrm{~km}$ $\mathrm{s}^{-1}$. However, what is striking is that shocks associated with low-latitude events are able to reach Ulysses at the South Pole of the Sun. The 2006 December $13 \mathrm{CME}$, evolved into a Magnetic Cloud driving a strong interplanetary shock, observed by near-Earth spacecraft (STEREO, ACE; Liu et al. 2008); see Section 3.3. Propagation of the solar wind data outward from 1 AU using an magnetohydrodynamic model showed Ulysses observed the same shock on December 17 as ACE/STEREO. This CME-driven shock was, thus, observed at both the Earth and Ulysses when they are separated by $74^{\circ}$ in latitude and $117^{\circ}$ in longitude, the largest shock extent ever detected in the space era (Liu et al. 2008).

Two separate enhancements are clearly distinguished in the $>50 \mathrm{MeV}$ ions and near-relativistic electron profiles in response to the X9.0 and X6.5 flares (Figure 2), with the second enhancement being more pronounced, possibly due to the very strong Moreton wave observed to flow out of the X6.5 flare leading to a more efficient acceleration of particles at these energies. For the later flares of this period, increases are only barely observed over the background level for the $>50 \mathrm{MeV}$ ion intensity and superposed on the decaying near-relativistic electron intensities. This is most likely due to the poorer magnetic connection of Ulysses to the flares during this period. At quiet times, the COSPIN/LET 8-19 MeV proton channel mainly responds to high-energy particles (galactic cosmic rays), but when the level of solar activity increases, it responds primarily to solar particles in the nominal energy range. One large gradual SEP event is observed in this energy channel. The onset and decay profiles of the event for $8-19 \mathrm{MeV}$ protons were relatively smooth (Figure 2). The event has a "clean" onset, occurring in a period nearly devoid of solar wind structures and with relatively low pre-event intensities. The onset time at Ulysses is defined as the time when the intensities exceed by more than $2 \sigma$ the background intensity, requiring that they continue to rise from then. Using 10 minute averaged data, we have found that the $8-19 \mathrm{MeV}$ proton intensities are observed to start increasing at 23:40 UT $\pm 0.166 \mathrm{hr}$ i.e., $\sim 13 \mathrm{hr}$ after the X9.0 flare. Using the solar wind velocity of $780 \mathrm{~km} \mathrm{~s}^{-1}$ measured by the Ulysses/SWOOPS experiment at the time of the event the Parker spiral magnetic field line connecting Ulysses to the Sun is computed to be $\sim 2.88$ AU long. The earliest expected onset time for protons propagating from the Sun along the field using the velocity of the highest energy ions contributing to the $8-$ $19 \mathrm{MeV}$ channel is calculated to be $\sim 1.98 \mathrm{hr}$ after the onset of the X9.0 flare. Comparison with the observations shows that the onset at Ulysses is considerably delayed. A slower rate of rise is observed after the occurrence of the X6.5 flare in the 8-19 MeV proton channel. No additional increase in the proton profiles in this channel was observed in response to the December 13 and 14 flares, possibly because it was masked by the already high pre-existing intensities (also the case for the near-relativistic electrons) and/or due to the poorer magnetic connection of Ulysses during this period. However, increases were observed in the lower energy profiles, with the lowest energy particles observed by HI-SCALE peaking at the time of passage of the shocks on DOY 345 and 351, suggesting a mixture of particles due to solar events and locally accelerated particles by the traveling interplanetary shocks.

Figure 3 focuses on the onset of the SEP event and shows differential proton intensities in the energy range $1.8-19 \mathrm{MeV}$ as measured by the COSPIN/LET instrument for the 2006 December 5-9 period. A velocity dispersion effect, with the higher energy particles starting to be detected earlier than the lower energy particles, is evident at the onset of the event 


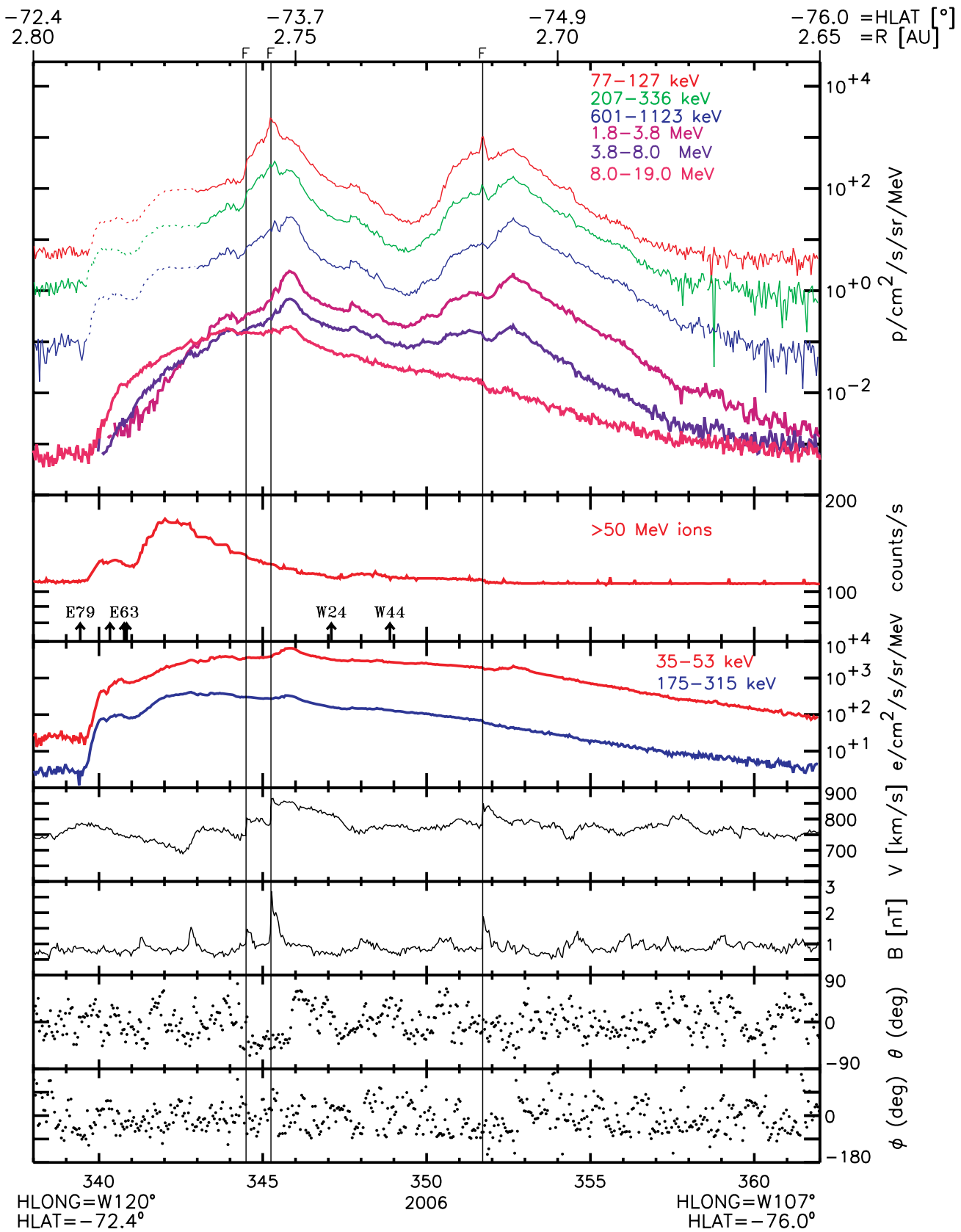

Figure 2. Overview of energetic particle observations as measured by the COSPIN/LET and HI-SCALE instruments onboard Ulysses, solar wind and magnetic field magnitude and directions in 2006 December. Solid vertical lines mark the arrival of interplanetary shock disturbances at Ulysses. Dotted traces in the HI-SCALE/ LEMS120 ion time intensity profiles indicate time intervals with possible electron contamination.

following the X9.0 flare. This is a noteworthy feature, not usually observed at COSPIN/LET energies up to $19 \mathrm{MeV}$ at the location of Ulysses ( $3 \mathrm{AU})$. It is reminiscent of particle events observed near the Earth. This is probably due to the fact that this is an isolated SEP event that occurred during a period of relatively quiet and stable conditions in the heliosphere. However, the observed time difference between the onsets at different energies by Ulysses was $\sim 1$ day, which is not consistent with the expected time delays for direct propagation along a Parker spiral, suggesting that the particles have undergone considerable diffusion.

\subsubsection{Composition Analysis}

Figure 4 shows heavy ion differential energy spectra for C, O, and $\mathrm{Fe}$ ions in the energy range $\sim 4-30 \mathrm{MeV} / \mathrm{n}$ as measured by the COSPIN/LET instrument, averaged over the period DOY $340-350$ of 2006. Errors bars are presented for all points for O and $\mathrm{C}$, whereas only one representative error bar is shown for $\mathrm{Fe}$. This period includes the two flare-related increases observed by the LET. No measurable heavy-ion fluxes were recorded by the LET beyond DOY 350. Also shown in Figure 4 are power-law fits to the data. The statistical errors have been used in the fit. The spectra for $\mathrm{C}$ and $\mathrm{O}$ show very similar spectral slopes over the measured energy range $(\gamma=-1.4 \pm 0.1)$, leading to an average $\mathrm{C} / \mathrm{O}$ ratio of 0.41 , typical of large SEP events. The Fe spectrum, which has been offset by a factor of 0.5 for clarity, appears to have a slightly steeper slope $(\gamma=-1.6 \pm 0.2)$, although the scatter in the individual points is large. Taking a simple event average, we find an $\mathrm{Fe} / \mathrm{O}$ ratio of $\sim 0.3$, indicating that the event is somewhat iron-rich. 


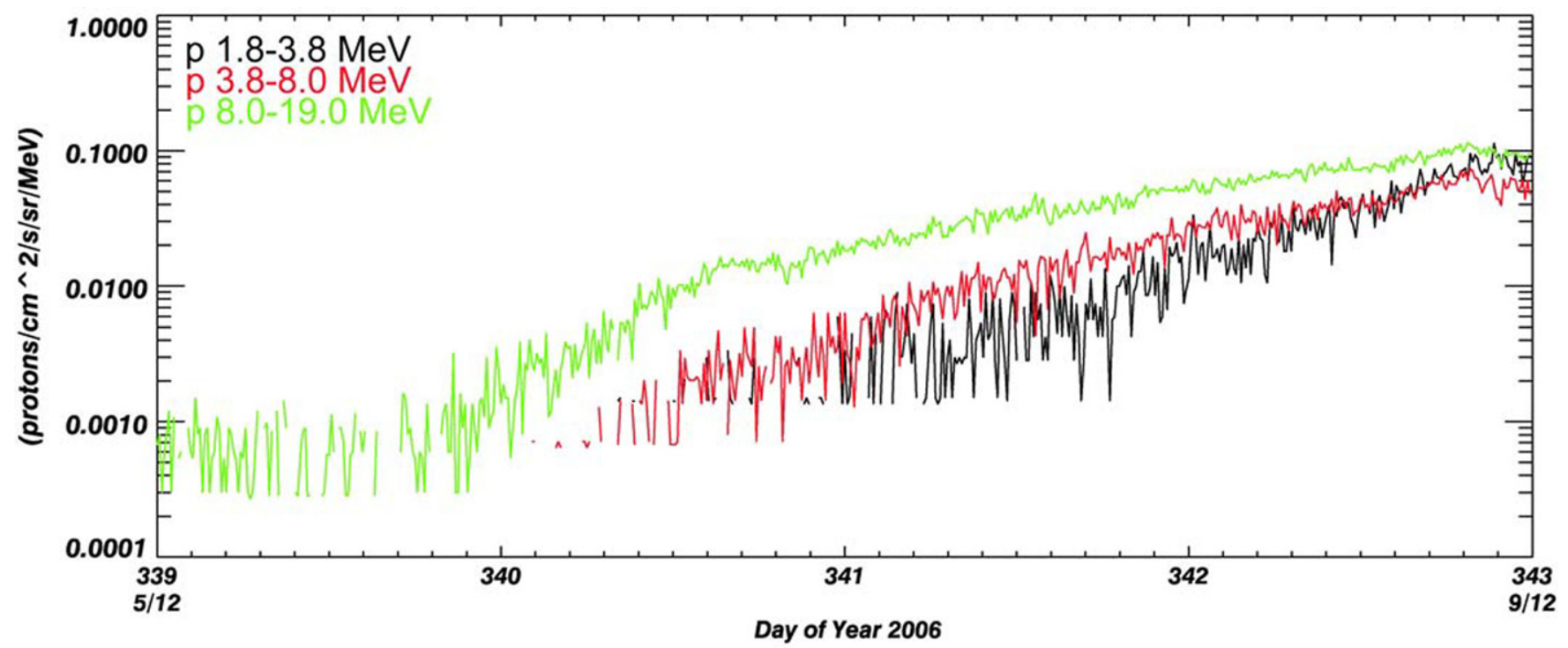

Figure 3. Zooming into the onset of the SEP event reveals a clear velocity dispersion effect is observed.

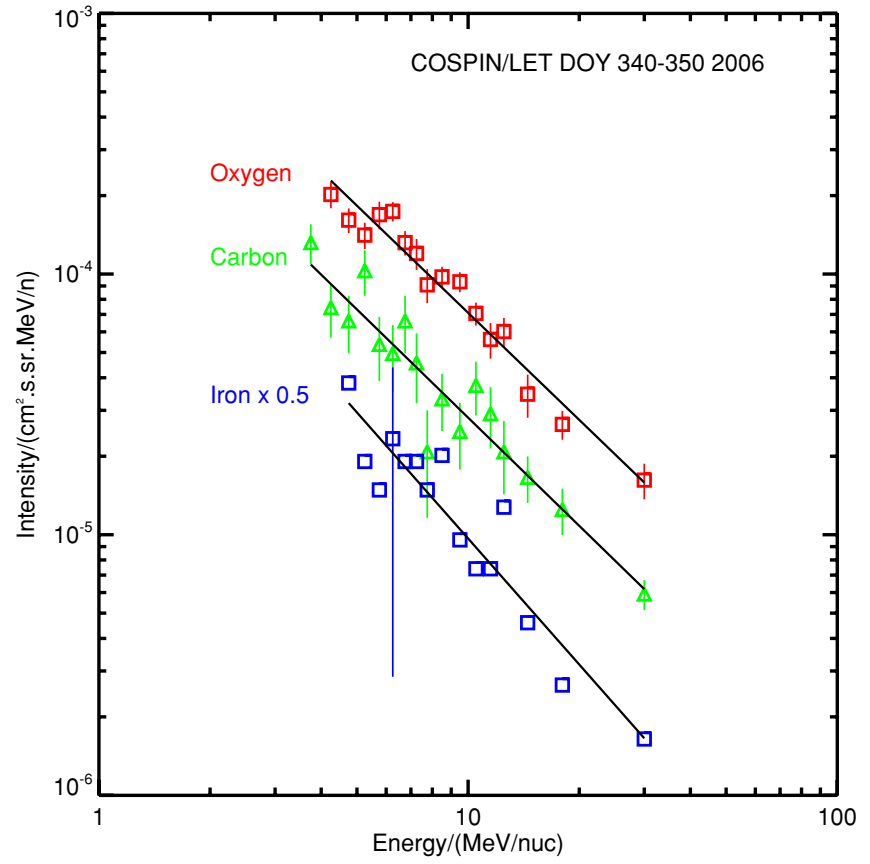

Figure 4. Heavy ion differential energy spectra for $\mathrm{C}, \mathrm{O}$, and $\mathrm{Fe}$ ions in the energy range $\sim 4-30 \mathrm{MeV}$ as measured by the COSPIN/LET instrument, averaged over the period 340-350, 2006. Power-law fits to the data are also shown.

\subsection{In-ecliptic 1 AU versus High-latitude Observations}

Stereos A and B were still near Earth and not well separated in 2006 December, so their observations were essentially identical. In Figure 5, differential proton intensities in the energy range $1.8-15 \mathrm{MeV}$ as measured by the LET experiment onboard the STEREO B spacecraft are shown for the period DOY 339-359, 2006. The spiky intensity increases observed on DOY 346 are associated with a radiation belt pass. The vertical arrows indicate the occurrence of the flares during this period (Table 1). The solid vertical lines correspond to the time of passage of three Forward shocks during this period identified by an abrupt increase in density, temperature, flow speed, magnetic field strength, and total pressure (not shown); see also (Liu

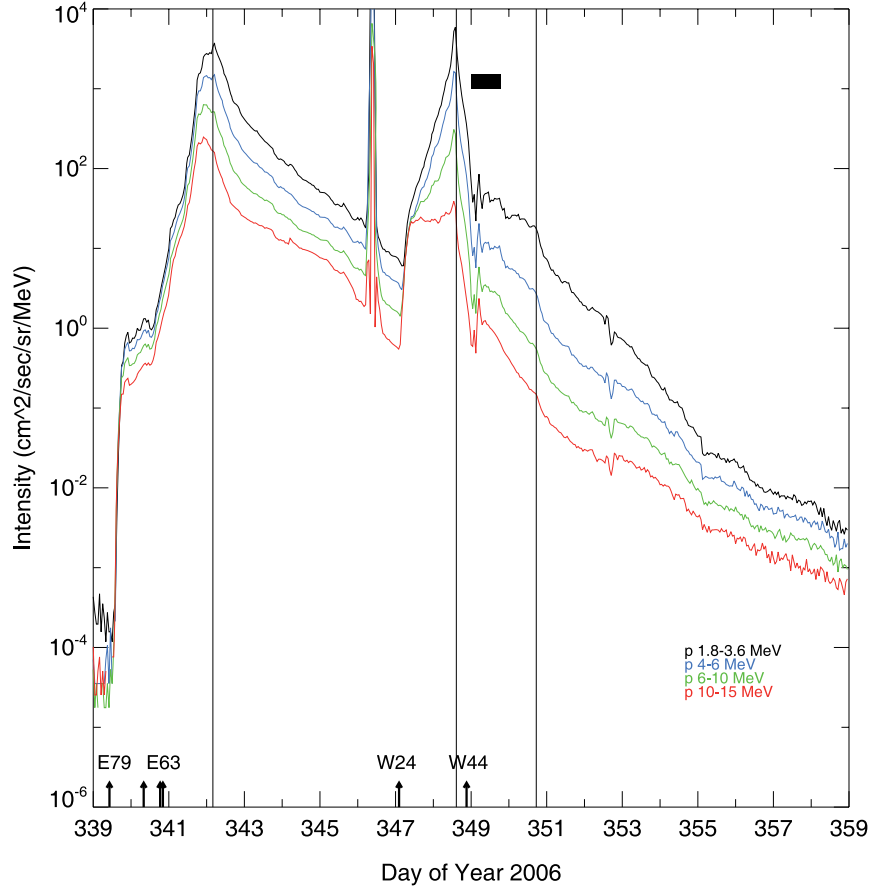

Figure 5. Differential proton intensities in the energy range $1.8-15 \mathrm{MeV}$ as measured by the LET experiment onboard STEREO B spacecraft for the period DOY 339-359, 2006. Solid vertical lines denote the passage of three forward shocks during this period. The black horizontal bar indicates the passage of a magnetic cloud over STEREO B.

et al., 2008). The black horizontal bar indicates the passage of a magnetic cloud as identified by Liu et al. (2008), based on bidirectional streaming electrons (BDEs), rotation of the field and low field variance. No plasma parameters were available from STEREO during this period. Complementary plasma parameters from $A C E$ reveal the passage of an interplanetary coronal mass ejection (ICME), with the magnetic cloud indicated by the STEREO BDEs being about the first half of the ICME interval (Liu et al. 2008).

Figure 6 shows hourly averages of $175-315 \mathrm{keV}$ magnetically deflected electron intensities as measured by the WART B 


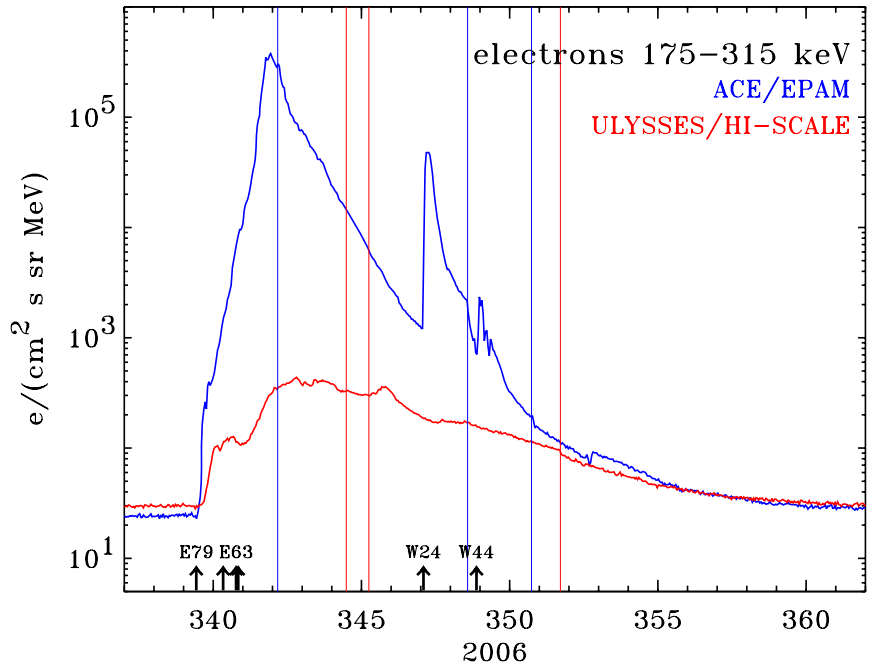

Figure 6. Comparison of near-relativistic electron intensities at high latitudes with those in the ecliptic plane near $1 \mathrm{AU}$ by the two identical instruments ULYSSES/HI-SCALE and ACE/EPAM, respectively.

detector head of the HI-SCALE experiment onboard Ulysses (red trace) and by the EPAM experiment, a reconfigured version of HI-SCALE onboard ACE at 1 AU (blue trace), during the 2006 December period. The intensities at the two locations achieved near equality only very late in the decay phase of the particle events, indicating the presence of the "reservoir effect" (Roelof et al. 1992), but only during that time interval. This effect was observed more prominently by Ulysses at high latitudes in the very disturbed interplanetary environment characteristic of solar maximum (e.g., McKibben et al. 2003).

Figure 7 presents $178-290 \mathrm{keV}$ electron intensities and their angular distributions as measured by the sunward looking telescope LEFS60 of the Ulysses/HI-SCALE and ACE/EPAM experiments during the onset of the event following the X9.0 flare on December 5. Pre-event background intensities have been subtracted to better visualize the onset of the event. Using 1 minute averaged data and defining the onset time of the event at $A C E$ and Ulysses, as the time when the intensities get $>2 \sigma$ above background and continue to rise from then on, the observed time delay between the arrival of the 178-290 electrons and the onset of the soft X-ray flare (vertical arrow in Figure 7) was $3.65 \pm$ $0.016 \mathrm{hr}$ for $A C E$ and $9.55 \pm 0.016 \mathrm{hr}$ for Ulysses. Using the solar wind velocity of $320 \mathrm{~km} \mathrm{~s}^{-1}$ measured by the $A C E /$ SWEPAM experiment (not shown) at the time of the event the Parker spiral

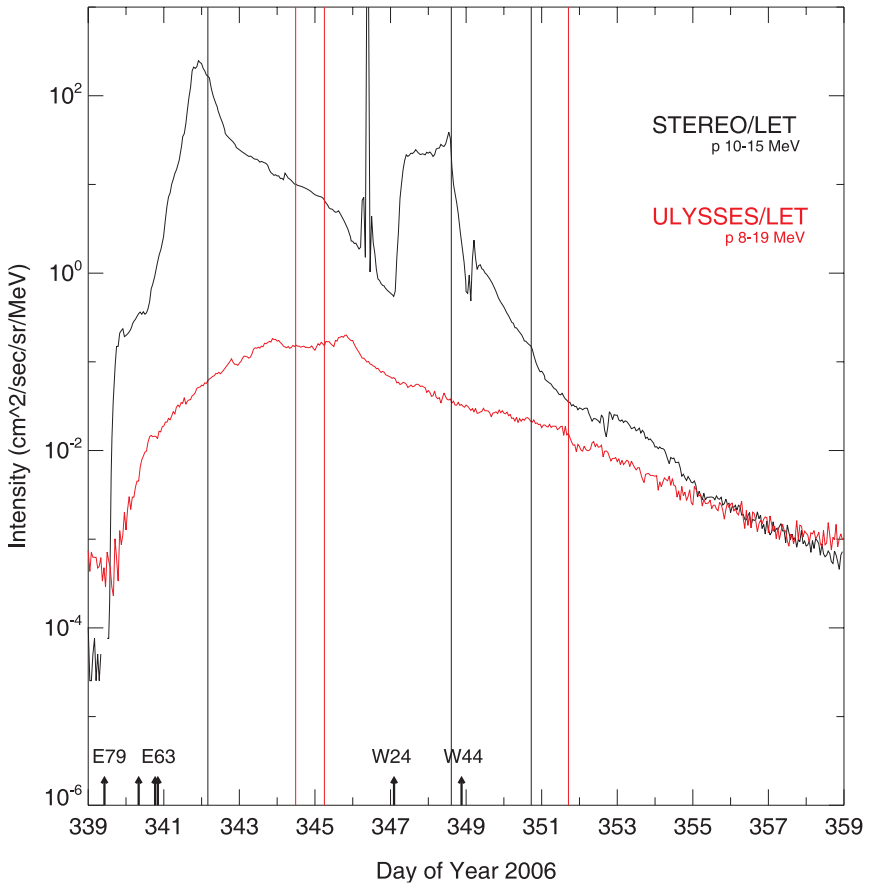

Figure 8. Hourly averages of proton intensities at similar energies as measured by the Ulysses/COSPIN/LET and STEREO/LET during the 2006 December period. Evidently, the rise time of the event at STEREO in response to the X9.0 flare is faster than at Ulysses.

magnetic field line connecting $A C E$ to the Sun is computed to be $\sim 1.23$ AU long. The earliest expected onset time for directly arriving near-relativistic electrons $(\sim 250 \mathrm{keV})$ propagating from the Sun along the field would be 14 minutes after the onset of the X9.0 flare. This corresponds to a not well-connected event as viewed from the Earth. The expected time delay between the arrival of these particles at Ulysses, travelling along a $\sim 2.88 \mathrm{AU}$ long Parker spiral magnetic field line connecting Ulysses to the Sun, and the onset of the soft X-ray flare would be 33 minutes, showing this is a considerably delayed onset. Pitchangle distributions at the onset of the event (insets at Figure 7) show a strong anisotropic flow away from the Sun at $A C E$, while at Ulysses near-isotropic electron angular distributions are observed after the onset of the event. The magnetic field in the fast solar wind is much more turbulent than in the slow solar wind, so propagation in the fast solar wind should be much more difficult (e.g., Sanderson 2004; Suess 2008) leading to

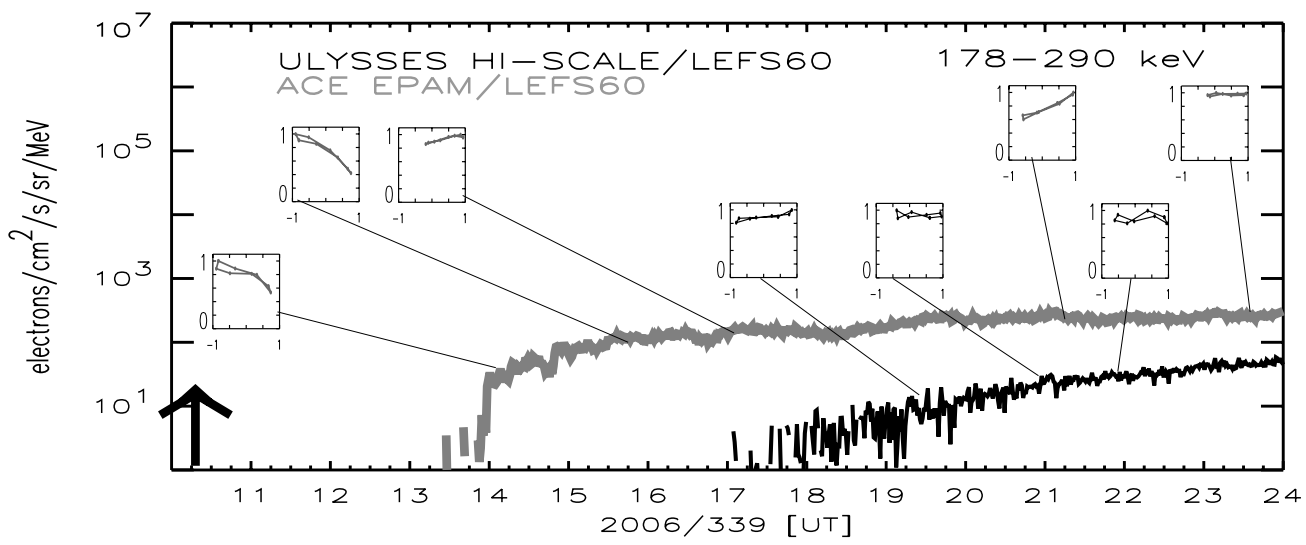

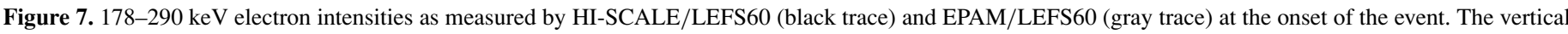

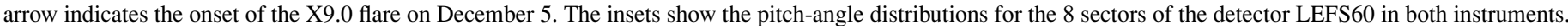


significant scattering of the particles. Figure 8 shows hourly averages of proton intensities at similar energy intervals as measured by the COSPIN/LET and STEREO/LET experiments onboard Ulysses (red trace) and STEREO B (black trace) during the 2006 December period. The rise time of the event at $A C E$ (Figure 6) and STEREO (Figure 8) in response to the X9.0 flare is faster than at Ulysses (although this corresponds to an eastern poorly connected event as viewed from the Earth). Thus, the onset of the SEP event at Ulysses suggests a more diffusive transport to high latitudes than to near-Earth spacecraft.

\section{DISCUSSION}

We report observations of unique large SEP events detected by Ulysses, during its third solar orbit, in 2006 December over the Sun's South Pole that occurred near solar minimum with a relatively quiet and stable interplanetary structure in the heliosphere. Throughout the first solar-minimum orbit of Ulysses only a very weak electron event observed at $\sim 73^{\circ} \mathrm{S}$ (Pick et al. 1995) was the highest latitude SEP event observed (Lario \& Pick 2008). The origin of the events in 2006 December was almost certainly the series of solar flares observed at low latitudes between December 5 and 14 .

The correlation between the large SEP events at near-Earth spacecraft (STEREO,ACE) and Ulysses suggests an easy access of SEPs at both low and high latitudes. Delayed onsets were detected for both the electrons and ions by the HI-SCALE and COSPIN/LET instruments onboard Ulysses. In order to explain the observation of large SEP events at high heliographic latitudes by Ulysses during its solar maximum orbit, Smith et al. (2001) suggested that SEPs access high latitudes via the complex magnetic field topology existing during solar maximum, where field lines originating at low latitudes may reach the polar regions. The relatively simple structure of the heliosphere near solar minimum as well as the observation of the 2006 December events when Ulysses was immersed in the southern high-speed coronal hole solar wind flow seems to exclude the possibility that magnetic field lines originating at low latitudes reached Ulysses. Very intense metric type II radio bursts were associated with the X-ray flares of this period, which provide evidence for strong propagating coronal shock waves at the Sun. Moreover, a rare Moreton wave flowing out of the X6.5 flare was observed. Moreton and EIT (Extreme-Ultraviolet Imaging Telescope) waves have been often associated with SEP events (Cliver et al. 1995; Torsti et al. 1998, 1999; Vainio \& Khan 2004; Mann 2006). The energetic particles observed as large SEP events over the South Pole of the Sun in 2006 December may therefore have been released when the propagating coronal waves reached highlatitude magnetic field lines connected to Ulysses. Calculations show that a coronal shock wave propagating away from the $\mathrm{X} 9.0$ flare at S07 would have to travel a distance of about $800,000 \mathrm{~km}$ across the solar disk to reach magnetic field lines at S73 connected to Ulysses. Assuming a speed of $\sim 1000 \mathrm{~km} \mathrm{~s}^{-1}$ for the coronal shock wave this implies a time delay of $\sim 13$ minutes, which would lead to a somewhat late injection of particles into high-latitude field lines connecting to the spacecraft. However, this delay apparently corresponds to a small fraction of the observed onset delays for both the ions and electrons.

An alternative explanation of the access of energetic particles at high latitudes in 2006 December may be the possibility of particle diffusion perpendicular to the mean magnetic field, able to spread the particles in the latitudinal direction, as originally suggested to explain the Ulysses observations during the Bastille Day 2000 event at high southern latitudes during solar maximum
(Zhang et al. 2003). Dalla et al. (2003) who analyzed nine highlatitude events observed by Ulysses are in agreement with the suggestion by Zhang et al. (2003). Note that for the Bastille Day 2000 event, a transient structure complicated the interpretation of the anisotropies observed (Sanderson 2004, 2005). Efficient cross-field diffusion close to the observer can be excluded during the rising phase of the 2006 December events since the particle flow directions were along the field and there was no evidence for any net flow across the field lines (Figure 7). Observations in the 2006 December events suggest that the particles propagated to high latitudes traveling along the magnetic field lines and not by crossing them. The particles were scattered significantly as they propagated outward, which explains their relatively slow onset and their small anisotropy, but despite this, any net flow direction was still along the local magnetic field line direction. On the basis of the observations available, however, crossfield diffusion closer to the Sun cannot be definitely excluded. These features are reminiscent of the four large SEP events Ulysses observed in fast solar wind during its second northern polar pass near solar maximum (Sanderson 2004, 2005; Lario et al. 2004). However, the 2006 December series of events that occurred during a period with relatively quiet and stable interplanetary conditions in the heliosphere, constitute a unique set of observations not seen previously during the entire Ulysses mission.

We are thankful to our HI-SCALE colleagues for their support and encouragement. Use of the Ulysses Data System is gratefully acknowledged. The CME catalog used to identify the solar origin of the SEP events is generated and maintained by NASA and the Catholic University of America in co-operation with the Naval Research Laboratory. SOHO is a project of international cooperation between ESA and NASA. We thank Manolis Zoulias for help in producing the final figures. The work at Caltech was supported by NASA under sub-contract SA271526309 from the University of California, Berkeley under NASA contract NAS5-03131.

\section{REFERENCES}

Balogh, A., et al. 1992, A\&AS, 92, 221

Bame, S. J., et al. 1992, A\&A, 92, 237

Cliver, E. W., et al. 1995, Proc. 24th Int. Cosmic-Ray Conf (Rome), 257

Dalla, S., et al. 2003, Geophys. Res. Lett., 30, 8035

Ebert, R. W., et al. 2009, J. Geophys. Res., A 114, 01109

Forsyth, R. J., Balogh, A., Smith, E., Murphy, N., \& McComas, D. 1995, Geophys. Res. Lett., 22, 3321

Gold, R. E., et al. 1998, Space Sci. Rev., 86, 541

Heber, B., et al. 2008, Proc. 30th Int. Cosmic-Ray Conf. (Merida), 217

Lanzerotti, L. J., et al. 1992, A\&AS, 92, 365

Lario, D., \& Pick, M. 2008, in The Heliosphere through the Solar Activity Cycle, ed. A. Balogh, L. J. Lanzerotti, \& S. T. Suess (Chichester, UK: SpringerPraxis), 151

Lario, D., et al. 2004, J. Geophys. Res., A 109, 01107

Liu, Y., et al. 2008, ApJ, 689, 563

Luhmann, J. G., et al. 2008, Space Sci. Rev., 136, 117

Malandraki, O. E., Marsden, R. G., Tranquille, C., Forsyth, R. J., Elliott, H. A., Lanzerotti, L. J., \& Geranios, A. 2007, J. Geophys. Res., 112, A06111

Malandraki, O. E., Marsden, R. G., Tranquille, C., Forsyth, R. J., Elliott, H. A., \& Geranios, A. 2008, Ann. Geophys., 26, 1029

Mann, G. 2006, in Geophys. Monogr. Ser. 165, Solar Eruptions and Energetic Particles, ed. N. Gopalswamy, R. Mewaldt, \& J. Torsti (Washington, DC: AGU) 221

McComas, D. J., Ebert, R. W., Elliott, H. A., Goldstein, B. E., Gosling, J. T. Schwadron, N. A., \& Skoug, R. M. 2008, Geophys. Res. Lett., 35, L18103

McKibben, R. B., et al. 2003, Ann. Geophys., 21, 1217

McKibben, R. B., et al. 2005, J. Geophys. Res., 110, A09S19

Mewaldt, R. A., et al. 2008, Space Sci. Rev., 136, 285 
Pick, M., Lanzerotti, L. J., Buttighoffer, A., Hoang, S., \& Forsyth, R. J. 1995, Geophys. Res. Lett., 22, 3377

Roelof, E. C., Gold, R., Simnett, G., Tappin, S., Armstrong, T., \& Lanzerotti, L. J. 1992, Geophys. Res. Lett., 19, 1243

Sanderson, T. R. 2004, in The Sun and the Heliosphere as an Integrated System, ed. G. Poletto \& S. T. Suess (Dordrecht: Kluwer), 113

Sanderson, T. R. 2005, in IAU Conf. Proc. 226, Coronal and Stellar Mass Ejections, ed. K. P. Dere, J. Wang, \& Y. Yan (Cambridge: Cambridge Univ. Press), 350

Simpson, J. A., et al. 1992, A\&AS, 92, 401
Smith, E. J., Balogh, A., Forsyth, R. J., \& McComas, D. 2001, Geophys. Res. Lett., 28, 4159

Suess, S. T. 2008, in The Heliosphere through the Solar Activity Cycle, ed. A. Balogh, L. J. Lanzerotti, \& S. T. Suess (Chichester, UK: Springer-Praxis), 252

Torsti, J. A., et al. 1998, Geophys. Res. Lett., 25, 2525

Torsti, J., Kocharov, L., Teittinen, M., \& Thompson, B. J. 1999, ApJ., 510 , 460

Vainio, R., \& Khan, J. I. 2004, ApJ, 600, 451

Zhang, M., et al. 2003, J. Geophys. Res., 108, 1154 